\title{
A Self-Injurious Behavior Causing Unusual Bilateral Gingival Recessions and Affecting the Periodontal Treatment: A Case Report
}

\author{
Diana Mostafa ${ }^{\mathrm{a}, \mathrm{c}}$, Pradeep Koppolu ${ }^{\mathrm{a}}$, Basel Tarakji ${ }^{\mathrm{b}}$
}

\begin{abstract}
Gingival recessions (GRs) can be multifactorial in its etiology. They occur either due to a mechanical influence or an inflammatory reaction in the gingival tissues. The cause should be diagnosed before taking any step in the treatment. The purpose of this case report was to highlight any adverse effect of the destructive ability of the psychological self-injurious behavior (SIB) to the gingiva and illustrate how such habits may affect the treatment success. In the reported case, a teenage patient had a constant habit of fingernails scratching that caused bilateral GRs in both mandibular canines. She admitted that this SIB was because of family problems. Usually, such persistent habit complicates the treatment; therefore, we planned for her psychological treatment first and then the periodontal surgical intervention using Alloderm ${ }^{\circledR}$ for root coverage. After 7 days of good healing and predictable results, the patient's careless behavior decreased the chances of the successful treatment.
\end{abstract}

Keywords: Fingernail inflicted gingiva; Gingival recession treatment; Self-injurious behavior; Acellular connective tissue allograft; Alloderm

\section{Introduction}

Recently, gingival recession (GR) has become a common problem in periodontal clinic. It is known to be due to the exposure of the root surface as a result of apical migration of the gingival margin from the cementoenamel junction (CEJ) [1]. It may appear to be clinically as localized or generalized. This can occur with or without loss of attached tissue [2].

Several such factors have been implicated in GR etiology:

\footnotetext{
Manuscript accepted for publication January 03, 2017

aDepartment of Preventive Dental Sciences, Al-Farabi Colleges, Riyadh, Kingdom of Saudi Arabia

bepartment of Oral Sciences and Diagnosis, Al-Farabi Colleges, Riyadh, Kingdom of Saudi Arabia

${ }^{\mathrm{c} C}$ Corresponding Author: Diana Mostafa, Department of Preventive Dental Sciences, Al-Farabi Colleges, Riyadh, Kingdom of Saudi Arabia.

Email: dr.dianamostafa@gmail.com
}

doi: https://doi.org/10.14740/jmc2719w anatomical, physiological, pathological and traumatic, and they may occur together coincidentally $[3,4]$. The common traumatic lesions in the oral cavity may be chemical, thermal or physical in nature [5-7]. However, the physical trauma is more severe, predominant and can implicate the deeper periodontal tissues.

One type of these physical injurious traumas is the selfinjurious behavior (SIB). This behavior refers to repetitive acts that cause physical damage to the individual without any intent to die but for purposes unacceptable socially [8,9]. It may occur as a solitary confined action or as a recurrent phenomenon, whether intentional or not. However, such behavior is relatively widespread manifestation of mental disorders, emotionally unbalanced personality, developmental disabilities, and some other syndromes [10,11]. It is usually due to psychic nature, mutilation, mental conflicts and emotions such as hate, jealousy, frustration, feelings of inferiority, etc. [12].

Sometimes the traumatic gingival injuries are called gingivitis artifacts [7]. These gingival injuries occur as a result of scratching, rubbing or picking the gingiva using the fingernail, or using abrasive objects $[6,13]$ where patients are aware of their damaging behavior but cannot stop it. Such cases seem to be much more common in children than in teenagers and adults and frequently in female patients with emotional disturbances [14]. The management of such cases is a challenge [15] in the dental clinic as the main cause is not dental in origin.

This report describes a case with SIB that caused an unusual self-inflicted gingival lesion. She was suffering from depression; her behavior caused bilateral recessions in both right and left mandibular canines. The periodontal treatment involved removal of the causative agent (the harmful behavioral habit), the potential ramifications of this habit are discussed, and the steps necessary to ensure and preserve the dental health were taken, including oral hygiene instructions and the scaling and root planning then the periodontal surgical procedures using the acellular connective tissue allograft $\left(\right.$ Alloderm $\left.^{\circledR}\right)$ were performed for root coverage.

\section{Aim}

Our purpose was to highlight the adverse effect of the destructive ability of the psychological SIB to the gingiva and illus- 


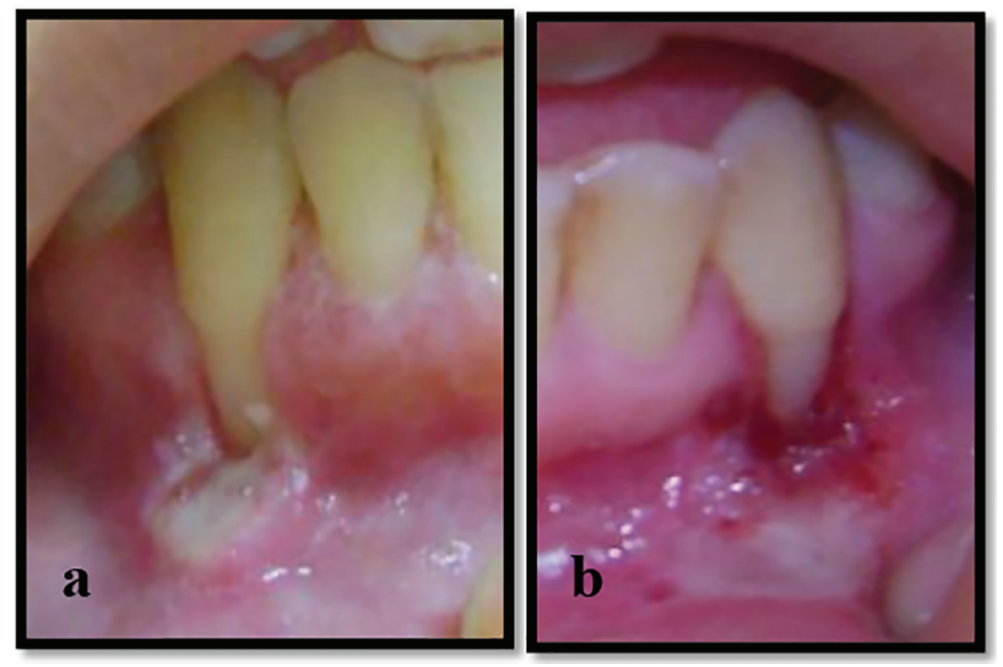

Figure 1. (a, b) Recession class II Miller's classification with ulcerations extending to alveolar mucosa related to the mandibular canines.

trate how these habits may affect the success of the treatment.

\section{Case Report}

A 15-year-old girl was referred to the Department of Periodontology Clinics, Faculty of Dentistry, Alexandria University, Egypt for evaluation and treatment of bilateral GR related to mandibular canines.

The medical and dental histories were taken and revealed that she was systemically and dentally healthy. She reported that the first time she noticed this recession was 8 months ago and it progressively worsened. The patient suffered from continuous burning pain in lower labial gingiva related to the lower canines. She also complained of pain and sensitivity in this area during drinking and also while coming in contact with food. She also reported that there were white membranes that were easily removed from the lower labial gingiva by her fingernails, resulting in red painful and bleeding areas.

Her parents stated that they had visited four physicians and the patient had taken systemic antibiotics without any improvement. Upon questioning, the patient concealed of doing any habits. Then latterly, as a result of a frank and friendly conversation with the patient, she admitted of scratching her gingiva by her fingernails for long periods since her parents started having the traumatic problems of their divorce procedures. The patient further stated that she became emotionally depressed and irritable. She believed that no one gave any attention to her and she felt neglected. This explained the clinical consequences of her harmful behavior.

\section{Periodontal evaluation}

On clinical examination, none of these clinical features existed: high frenal attachment, traumatic overbite and bruxism secondary to premature tooth contact. The gingiva in both regions had an ulcerated appearance of edematous, reddish margins extending to alveolar mucosa. There were white, yellowish membranes that were slightly removed by gauze leaving very painful erythematous bleeding areas. Minimal plaque deposits were noticed (Fig. 1).

The periodontal evaluation revealed Miller's class II [16] GRs on the facial surface of both mandibular canines, extending $9 \mathrm{~mm}$ apical to the CEJ in the left mandibular canine and $8 \mathrm{~mm}$ apical to the CEJ in right mandibular canine, a narrow zone of attached gingiva $(1 \mathrm{~mm})$ and no loss in height of the mesial and distal interdental papillae. There was no GR associated with adjacent teeth (Fig. 1).

On radiographic examination using periapical radiographs, no evidence of proximal bone loss in the mesial and distal aspects of the affected teeth was found.

\section{The causative habit}

The patient explained that her habit was of using her index fingernails of both hands, pushing the mandibular canines gingiva in a downward direction at the same time. She added that she liked to scratch the mucosa beneath the canines until bleeding.

\section{Treatment consideration}

After the diagnosis of self-inflicted gingival lesions and the patient's harmful behavior, the patient and her family were taught and became aware of the squeals of her self-inflicted injury. She comprehended the effect of her scratching habit and how much damage it would cause to the gingiva and she was also convinced to discontinue this habit. She was asked to trim her fingernails, replace her old toothbrush and avoid the use of abrasive pastes to evade any harmful effect on the gingival tissue. She was then referred to a psychiatrist for evaluation, discovering, treatment and elimination of this behavior. The behavioral 


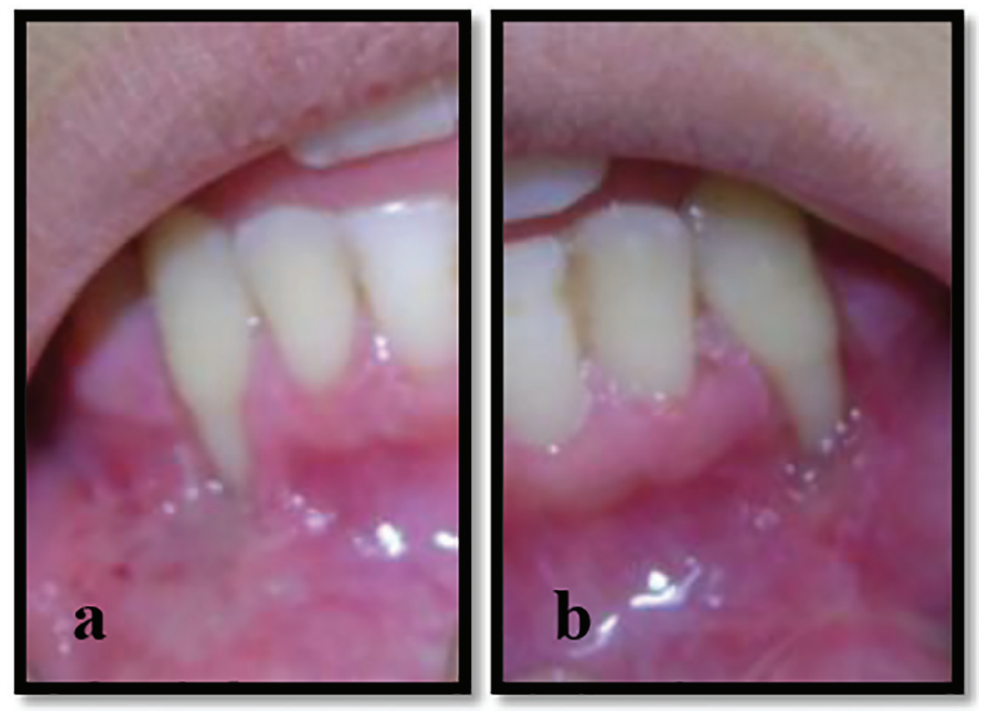

Figure 2. $(a, b)$ Ulcerations and inflammation were resolved after periodontal treatment.

treatment included awareness training to sensitize the patient to the occurrence of this behavior, teaching the patient how to face and deal with her apprehensive attitude, giving alternatives rather than nail scratching and follow-up social support.

The first steps in the periodontal treatment of the GR were taken. Scaling, root planing and polishing were done. Oral hygiene instructions were given, including the flossing and the brushing procedures, using an extra soft toothbrush with a technique starting apically on the gingiva and gently sweeping coronally with the toothbrush. Chlorhexidine gluconate $0.12 \%$ rinse was prescribed twice daily for 2 weeks to decrease the mucosal inflammation and infection. The patient started to improve and her pain decreased considerably (Fig. 2a, b).

After 4 months of progressive psychological treatment and periodical dental re- evaluation visits, it was affirmed that the patient was in a stable psychological state and willing to have root coverage surgery. We started the periodontal surgical treatment for the purpose of restoring the esthetic appearance of the GR and increasing the attached gingiva zone. We decided to use the acellular connective tissue allograft (Alloderm ${ }^{\circledR}$ ) for root coverage to avoid palatal graft donor surgical site.

Before incision, a very careful root planning was done to the root surface by Gracey's curettes to remove plaque, calculus, and soft tooth structure. Any gross irregularities and prominent root eminences were smoothed by fine diamond bur and the sites were rinsed with a sterile saline solution. The exposed roots were conditioned by burnishing it with tetracycline (100 $\mathrm{mg} / \mathrm{mL}$ for $4 \mathrm{~min}$ ). Two split mucoperiosteal flaps were done by scalpel blade no. $15 \mathrm{C}$ (Hu-Friedy). Horizontal incisions were made at the level of CEJ corresponding to the base of adjacent papilla, and then vertical incisions were placed from line angles of neighboring teeth extending apically beyond the mucogingival junction in trapezoidal design (Fig. 3a, b). The acellular dermal matrix allograft (Alloderm) was dehydrated with a sterile saline for $10 \mathrm{~min}$ according to the manufacturer's guidelines. The alloderm was trimmed and adjusted to fit the recipient areas at the CEJ.

First, interrupted sutures were performed to secure the allograft in its position at the base of the mesial and distal papillae using resorbable 5-0 chromic gut sutures in an interrupted
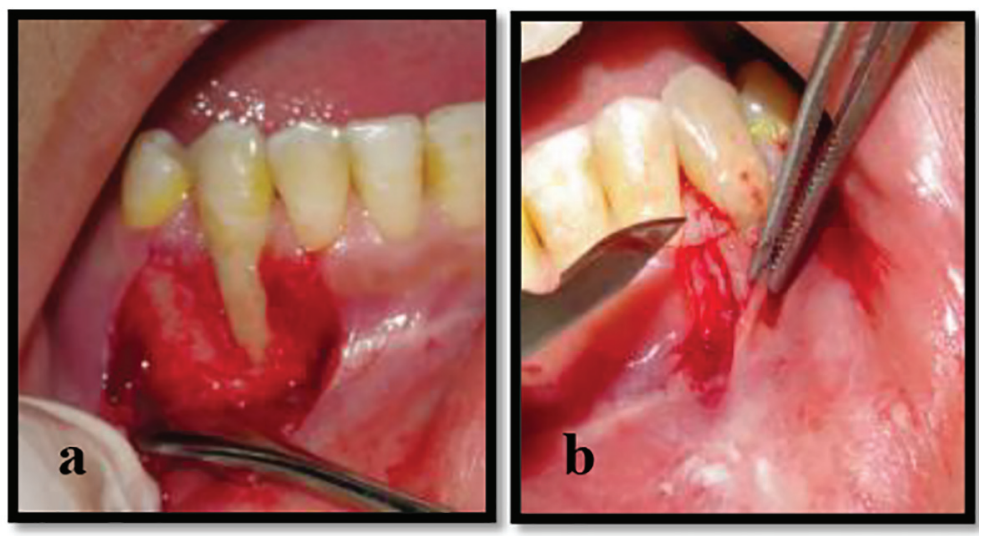

Figure 3. (a, b) The split mucoperiosteal flaps were done from the base of interdental papillae of both sides. 

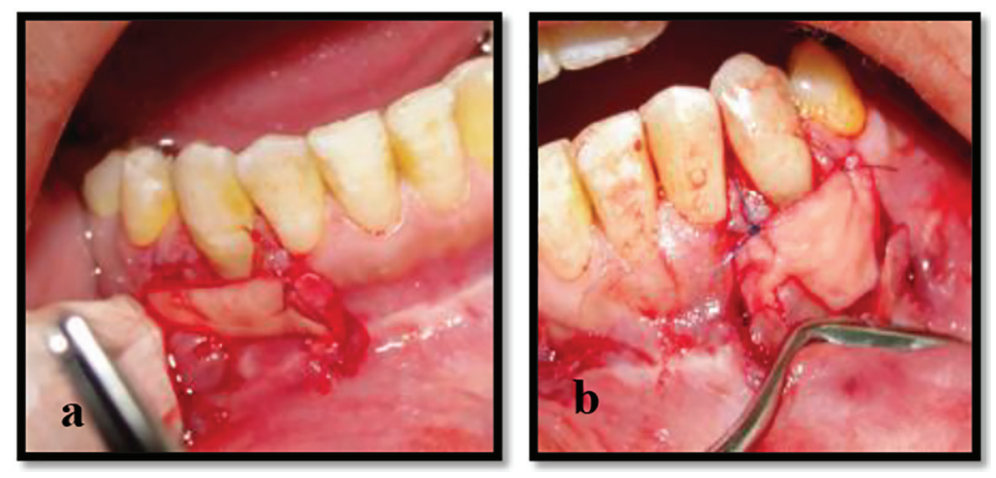

Figure 4. $(a, b)$ The application and suturing of Alloderm to the base of papillae of the left and right mandibular canines.

configuration. The intact papillae helped the Alloderm to be stable in the surgical sites (Fig. 4a, b) and the flaps covered the entire graft and were sutured tension free. Periodontal dressing was applied to surgical sites to protect the wound and increase stability of the surgical site during the healing process.

Amoxicillin, $500 \mathrm{mg}$, three times a day for 7 days was prescribed and analgesics were prescribed as needed. A gentle ice pack was applied immediately after surgery on a 20-min intermittent basis for the first $24 \mathrm{~h}$ on both sides. She was advised to eat a soft diet for the next 2 weeks. After 7 days, the periodontal dressings were removed. The patient was recommended to use cotton applicators saturated with chlorhexidine gluconate $0.12 \%$ on the surgical sites and avoid scratching or trauma to the surgical sites. Excellent results were predicted as the patient was so co-operative and had the willingness to get the periodontal surgery done.

\section{Clinical observation and results}

After 7 days postoperatively, the patient was asked to come every week for re-evaluation and irrigation of the surgical sites. The healing was progressively good and the gingival appearance started to become harmonious with the other teeth showing the maturation of Alloderm graft (Fig. 5a, b). Then, the surgical site was covered again with periodontal pack to avoid any risk of infection.

At the 1-week later review, she came back to our clinic with unexpected results; there were no gingiva coverings on the facial part of canine's roots. The final clinical picture was the same as of the first one before the periodontal surgery.

Upon asking about the cause for her baleful attitude towards the root coverage treatment and its healing, she gave a wondering expression as if she did not know what had happened to the surgical site. She then admitted that she had removed the periodontal pack and started the SIB again. She did not feel any guilt. The patient was again shown the damage she had caused to the gingiva and requested her to stop her injurious behavior and referred her to the psychiatrist for advanced retreatment. We advised the patient to complete the follow-up visits for her re-evaluation. Unfortunately, she did not continue the follow-up visits and refused her psychiatric re-treatment. Consequently, the failure of the whole periodontal treatment could be predicted.

Regarding the psychological explanation, the main cause of the patient's harmful habit was due to her volatile emotions towards her parent's conflicts and her weak personality to face such problems, and self-harm became a response to her profound emotional pain that could not be resolved in a more functional way. It was concluded that the relapse and continu-
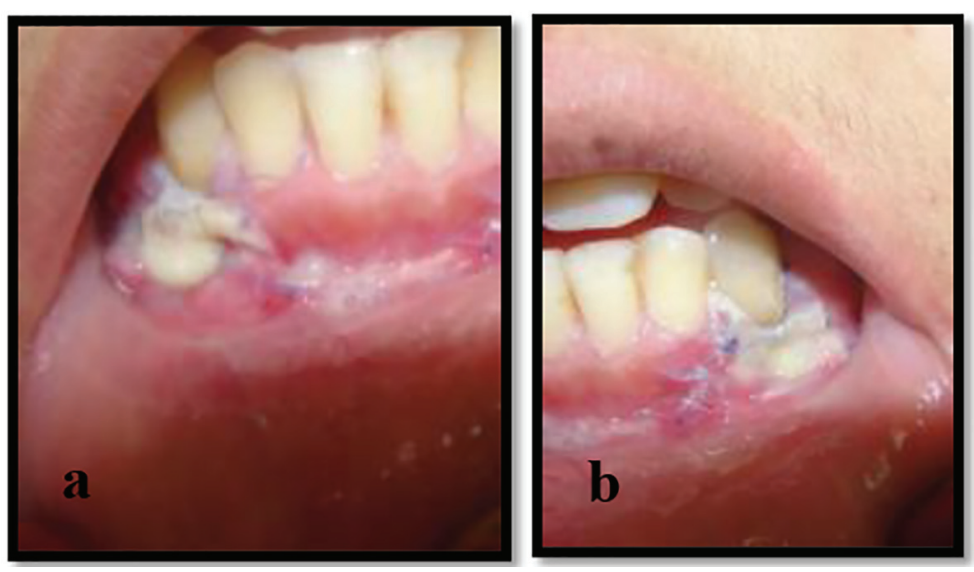

Figure 5. (a, b) Normal postoperative healing, whitish color was related to epithelial sloughing and homogenisty indicated good expections. 
ity of this habit were due to her excessive attention seeking and the addiction of causing trauma.

\section{Discussion}

The SIB is usually of a psychic nature, originating from mental or emotional disturbance. The most common ones are stress, anxiety and depression. However, this behavior has been recorded intensely in the past several decades. Although neither a hard nor a soft oral tissue is excluded from the harmful effects of these psychological influences, the gingiva is the most affected tissue [17].

The treatment of SIB is very problematic as its management includes both the dental and the psychological approaches. This type of behavior is more persistent with the conventional treatment as it is usually associated with an emotional disturbance [5]. It is seriously influenced by the patient's ability to discontinue his behavior and to make a conscious obedience of his treatment.

Also, there are no standard techniques to prevent or treat self-inflicted injuries. The treatment is planned according to the case's circumstances [18]. The lesions may persist longer and may relapse after curing [19]. Therefore, several challenges need to be overcome; otherwise, the treatment could be complicated by their negative attitude and the lack of their compliance.

In this reported case, the teenage girl had a compulsive habitual fingernail scratching, which caused GRs in both mandibular canines. This case has highlighted and broadened the causative spectrum of GR. However, if the periodontal findings were presented with local factors (e.g., plaque, calculus, developmental teeth defect), the case could have been misdiagnosed.

After 7 days of good healing outcomes, her incessant injurious habitude despite her knowing of its harmful effect on the gingiva has affected the case in stabilization and interrupted the gingival healing, resulting in the failure of the whole periodontal treatment.

\section{Conclusion}

Before treatment of any oral mucosal lesion, it is advisable to recognize the main cause of the lesion without excluding any suspected reason to avoid persistence or recurrence of the lesion.

Taking a proper patient history, proper periodontal evaluation, enrollment, all factors of the GR, awareness regarding the incidence of these habitual factors even in the presence of other obvious etiology is mandatory, otherwise misdiagnosis and mistreatment will be the consequence. Psychotherapeutic counseling and early detection of the cause is the first step for management of periodontal patients with SIB. The severity of gingival artifacts is related to degree and periods of stress and anxiety. The prognosis and treatment success are completely dependent on their compliance, willing attitudes and conscious change of their behavior.
The psychological treatment success totally affects the prognosis and achievements of periodontal treatment in such cases. Therefore, the SIB is considered as one of the risk factors in periodontal clinic.

\section{Conflicts of Interest}

None.

\section{Consent}

Informed consent and verbal approval were obtained from the patient's parents. Full explanation was offered to the patient and patient's parents before any treatment procedures.

\section{References}

1. Pradeep K, Rajababu P, Satyanarayana D, Sagar V. Gingival recession: review and strategies in treatment of recession. Case Rep Dent. 2012;2012:563421.

2. van Palenstein Helderman WH, Lembariti BS, van der Weijden GA, van 't Hof MA. Gingival recession and its association with calculus in subjects deprived of prophylactic dental care. J Clin Periodontol. 1998;25(2):106111.

3. Susin C, Haas AN, Oppermann RV, Haugejorden O, Albandar JM. Gingival recession: epidemiology and risk indicators in a representative urban Brazilian population. J Periodontol. 2004;75(10):1377-1386.

4. Tugnait A, Clerehugh V. Gingival recession-its significance and management. J Dent. 2001;29(6):381-394.

5. Spencer RJ, Haria S, Evans RD. Gingivitis artefacta - a case report of a patient undergoing orthodontic treatment. Br J Orthod. 1999;26(2):93-96.

6. Greene PR. An unusual self-inflicted gingival injury. $\mathrm{Br}$ Dent J. 1994;177(1):23-24.

7. Dilsiz A, Aydin T. Self-inflicted gingival injury due to habitual fingernail scratching: a case report with a 1-year follow up. Eur J Dent. 2009;3(2):150-154.

8. Nock MK. Why do People Hurt Themselves? New Insights Into the Nature and Functions of Self-Injury. Curr Dir Psychol Sci. 2009;18(2):78-83.

9. Feldman MD. The challenge of self-mutilation: a review. Compr Psychiatry. 1988;29(3):252-269.

10. Herpertz S. Self-injurious behaviour. Psychopathological and nosological characteristics in subtypes of self-injurers. Acta Psychiatr Scand. 1995;91(1):57-68.

11. McDonald RE, Avery DR. Dentistry for the Child and Adolescent. 7th edition. Mosby. 2000; p. 467-469.

12. Limeres J, Feijoo JF, Baluja F, Seoane JM, Diniz M, Diz P. Oral self-injury: an update. Dent Traumatol. 2013;29(1):8-14

13. Stewart DJ. Teething, an unusual response. A case of gingivitis artefacta minor in an infant. Br Dent $\mathrm{J}$. 1982;152(3):93-94. 
14. Pattison GL. Self-inflicted gingival injuries: literature review and case report. J Periodontol. 1983;54(5):299-304.

15. Lucavechi T, Barberia E, Maroto M, Arenas M. Selfinjurious behavior in a patient with mental retardation: review of the literature and a case report. Quintessence Int. 2007;38(7):e393-398.

16. Miller PD, Jr. A classification of marginal tissue recession. Int J Periodontics Restorative Dent. 1985;5(2):8-13.
17. Sumaiya Arfin, Shazra Tasneem, Saima Yunus Khan, M K Jindal. Self-inflicted palatal soft tissue injury: a case report. American Journal of Research Communication. 2014;2(10):153-159.

18. Ayer WA, Levin MP. Self-mutilating behaviors involving the oral cavity. J Oral Med. 1974;29(1):4-7.

19. Hoffman HA, Baer PN. Gingival mutilation in children. Psychiatry. 1968;31(4):380-386. 